\title{
Synthesis, characterization and physicochemical studies of copolymers of aniline and 3-nitroaniline
}

\author{
Umesh Somaji Waware $^{1}$ - A. M. S. Hamouda ${ }^{1} \cdot$ Dipanwita Majumdar $^{2}$
}

Received: 12 November 2018 / Revised: 10 September 2019 / Accepted: 12 September 2019 /

Published online: 9 October 2019

(c) The Author(s) 2019

\begin{abstract}
Polyaniline (PA), the versatile conducting polymer, owing to its tunable optoelectronic properties, facile preparation methodology and reversible redox behavior, has elicited much interest among current researchers, particularly in the fields of energy generation storage devices, protective coatings and electrochemical sensors. However, its commercialization has been much restricted due to low solution processability and thermal stability. Recent studies reveal that the above-mentioned challenges can effectively be addressed by copolymerization of PA with suitable components. In addition, the properties of copolymers could be modified and tuned by varying the monomer ratios. Thus, the present work is concerned with the fabrication of poly(aniline-co-3-nitroaniline) with varying compositions obtained by in situ oxidative copolymerization of aniline and 3-nitroaniline by altering the molar ratio of monomers. Optimization of the physicochemical properties such as UV-visible absorption, solubility, thermal stability, electrical conductivity and dielectric signatures, particle size and morphology was achieved by varying the composition of monomeric substituents in these copolymers. Smoother morphology of the copolymer films was revealed by morphological studies via AFM technique and supported by particle size distribution study. The physicochemical trends demonstrated that proper proportions of nitro $\left(-\mathrm{NO}_{2}\right)$ group in the polymer chain are essential to achieve desired optimal physicochemical properties. Therefore, copolymers are ideally appropriate for multifaceted applications and would promote wider usage of conjugated polymers in various fields of organic-based optoelectronic as well as energy storage devices in the near future.
\end{abstract}

Keywords Polyaniline Copolymerization · Poly(aniline-3-nitroaniline) · Physicochemical properties $\cdot$ Processability

Dipanwita Majumdar was formerly at Department of Chemistry, Barasat Govt. College, Barasat, 700124, West Bengal, India.

Electronic supplementary material The online version of this article (https://doi.org/10.1007/s0028 9-019-02957-y) contains supplementary material, which is available to authorized users.

Extended author information available on the last page of the article 


\section{Introduction}

The conjugated polymers on account of their unique electrical, optical and chemical properties have emerged as a novel class of synthetic materials ideally suitable for a variety of technological applications such as solar cells [1-3], sensors [4], electromagnetic shield coatings [5], OLEDs and in energy storage devices [6, 7]. Among these different conjugated polymers, polyaniline (PA) and its derivatives have been studied extensively ever since its' discovery through the pioneering works by renowned MacDiarmid group [8, 9]. Polyaniline is popular owing to its ease of synthesis in aqueous media, reversible proton doping, highly tunable electrical conductivity, relative environmental stability and advanced optoelectronic properties [8-10]. Different micro- and nanostructures of PA have displayed versatile applications in diversified fields of organo-electronics [11-15].

However, the foremost problem in applying in using PA pristine form is its poor solution processability. The polymer is exceedingly less soluble in common solvents and suffers degradation prior to melting on thermal treatment $[16,17]$. Many researchers have opted various way outs in overcoming the above problems by generating polyaniline-based composites or blends with different metal oxides; various nanocarbon forms like fullerenes, CNTs, graphenes and others can show better stability, processability and mechanical strength along with superior optoelectronic properties [18-20]. These fabricated materials have their own benefits as well as limitations, but still large-scale cost-effective strategies for effective designing of these composites as required for desired applications are still on continuous search [21-28].

Copolymerization technique offers a simple, cost-effective way of improving the processability of polymers to an appreciable extent. It greatly enhances the scope for polymer scientists and considerably improves the properties of the derivatives compared to that of the homopolymers [29]. Moreover, it yields materials with uniform composition and the properties can be modified plainly by adjusting the ratio of concentration of starting monomers [30, 31]. Literature survey reveals that copolymerization procedure at present has become an effective way out for polymer engineering especially at the molecular level $[32,33]$. The copolymerized polyaniline family is very challenging because of its tunable conductivity and solubility in polar organic solvents that depend not only on the oxidation state but also on the degree of protonation and nature of dopants $[34,35]$. The properties of the poly (substituted anilines) like toluidine, anisidine and halogen-substituted anilines, etc., significantly vary with the nature of substitution (R) which may be either electron-withdrawing or electron-donating groups. Electron-withdrawing groups generally tend to reduce the electron density in aniline moiety, while electron-donating groups enhance the electron density in the phenyl ring of aniline. The present authors have also reported many works based on physicochemical properties study on copolymers of aniline with halogen-substituted anilines in the recent past [36-38]. The resultant copolymers exhibited impressive results with respect to solubility, viscosity, magnetic properties, although they possess very low electrical conductivity for practical utility. 
Thus, attempt for advanced materials is always in the continuous run! [39, 40]. In this regard, it is worth mentioning that an ab initio study employing DFT was carried out to elucidate the effect of polyaniline backbone functionalization on its properties in the recent past [41]. They reported that the energy characteristics of HOMO and LUMO levels of the polymers get affected due to functionalization, as a result of which the polarity also gets modified depending on the nature of functional moieties. Thus, functionalizing polymer backbone with proper functional groups in correct proportions may be an effective approach to achieve tailored conductive polymers with desirable properties [41].

It is well documented that poly3-nitroaniline (P-3-NA) is easily obtainable, and fairly stable in neutral, acidic or alkaline solutions unlike PA and is classified as "not readily biodegradable" with "low bioaccumulation potential" [42]. Copolymers of aniline with 3-nitroaniline, i.e., poly(aniline-co-3-nitroaniline), have been readily synthesized in various molar ratios of comonomers by chemical and electrochemical polymerization. It is true that both aniline and nitroanilines are reported to be toxic, but polymerization with aniline has been opted as an efficient method for removal of m-nitroaniline from wastewater [43]. The copolymerized products due to lower solubility owing to the larger size of the polymer compared to monomers are less toxic/carcinogenic as well [44]. Thus, this copolymerization method may be considered as an effective green step for proper usage and disposal of toxic by-products obtained from various dyes and textile industries.

Studies revealed that the kinetics of copolymerization varies with monomer concentration. The copolymerization rate decreased with an increasing ratio of 3 -nitroaniline in the monomer [45]. Recent investigations reported that $-\mathrm{NO}_{2}$ group substitution into PA chains modifies the electronic and vibrational spectra of the resultant macromolecule [46]. Lately, it has been established that both the polymerization rate and yield of poly (nitroaniline) gets enhanced in the presence of trace amount of aniline acting as an initiator/promoter for the reaction [47, 48]. Nevertheless, systematic investigation regarding variation of physicochemical properties by introducing controlled proportions of strong electron-withdrawing such as $-\mathrm{NO}_{2}$ groups as substituents in PA chain segments yet remains unexplored. Thus, in this work, poly (aniline-co-3-nitroaniline) was prepared by simple chemical oxidative polymerization procedure with varying monomeric compositions and the resultant copolymers are characterized using sophisticated instrumental techniques such as UV-visible spectroscopy, FTIR spectroscopy, TGA, AFM imaging, particle size analyses for better understanding of their characteristics to be employed in various organo-electronics applications. The current study emphasizes on tuning the physicochemical properties such as UV-visible absorption, solubility, thermal stability, electrical conductivity, particle size distribution and surface smoothness of the resulting copolymer compositions so that, in the near future, such copolymers may claim substantial implication for their contribution in the various challenging fields of science and technology. 


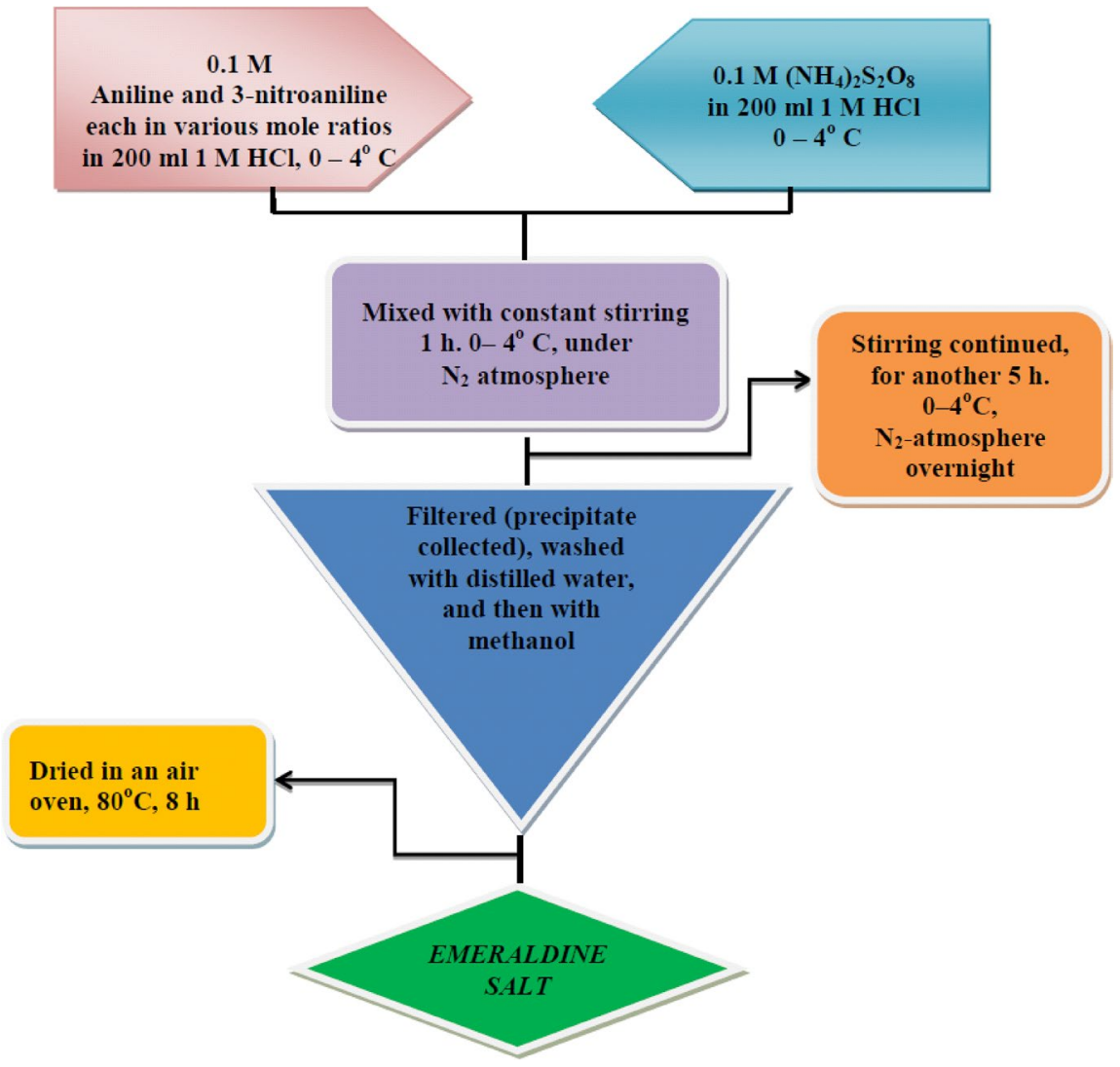

Scheme 1 Steps involved in the copolymerization process

\section{Experimental section}

\section{Chemicals and materials}

Aniline (99.5\%), 3-nitroaniline (98.0\%) and ammonium peroxodisulphate (98.0\%) were purchased from the Sigma-Aldrich Chemical Company and used as supplied. Analytical grade hydrochloric acid $(\mathrm{HCl})(95.0 \%)$ and spectroscopic grade $\mathrm{N}$-methylpyrrolidone (NMP) (99.5\%) and methanol (MeOH) (99.9\%) were used as received. Double-distilled water was used in the preparation of aqueous solutions in all cases.

\section{Synthesis of polyaniline derivatives}

The polyaniline (PA) and poly(3-nitroaniline) (P-3-NA) homopolymers were synthesized by oxidation of aniline and 3-nitroaniline separately with ammonium peroxodisulphate in $1 \mathrm{M}$ hydrochloric acid according to modified procedure reported $[39,40]$ and schematically represented in Scheme 1 . In a typical reaction, aniline 
(20 mmol, $1.86 \mathrm{~g}$ ) was added to $200 \mathrm{~mL}$ of $1 \mathrm{M} \mathrm{HCl}$ and the mixture was cooled to $0-4{ }^{\circ} \mathrm{C}$ in an ice bath. An ice-cold solution of ( $\left.20 \mathrm{mmol}, 4.56 \mathrm{~g}\right)$ ammonium peroxodisulphate, dissolved in $200 \mathrm{~mL}$ of $1 \mathrm{M} \mathrm{HCl}$, was then added dropwise into the precooled monomer solution with constant stirring under nitrogen atmosphere. Maintaining of low $\mathrm{pH}(\sim 1 \mathrm{M} \mathrm{HCl})$ medium during polymerization process promotes head-to-tail coupling of aniline monomers to form the desired linear polymers with head-to-tail coupling and helps in avoiding tail-tail coupling leading to undesired benzidine-type segment formation [49]. The reaction mixture was further stirred for another $5 \mathrm{~h}$ and maintained at room temperature for $24 \mathrm{~h}$ which ultimately resulted in a dark green precipitate of the polymer in emeraldine salt form (PA-HCl). The resultant polymer was then filtered, washed with small volumes of $1 \mathrm{M} \mathrm{HCl}$ and dried in an air oven for $8 \mathrm{~h}$ at $80{ }^{\circ} \mathrm{C}$. Likewise, poly (3-nitroaniline), (P-3-NA) was obtained by oxidative polymerization of 3-nitroaniline using ammonium peroxodisulphate, treated with similar procedure as stated above, that ultimately produced a brown colored powder.

Copolymers of aniline and 3-nitroaniline (PA-co-3-NA) were obtained by chemical oxidative coupling of the monomers in $1 \mathrm{M} \mathrm{HCl} \mathrm{using} \mathrm{ammonium} \mathrm{per-}$ oxodisulphate in a similar procedure as stated above. To the aqueous solution of aniline and 3-nitroaniline in $1 \mathrm{M} \mathrm{HCl}$, ammonium peroxodisulphate as an oxidant (molar ratio of monomers to oxidant 1:1) was added to carry out oxidation to obtain the copolymers of different composition; the mole fraction of 3-nitroaniline in the reaction mixture was varied from 0.2 to 0.8 . The precipitate formed of the as-synthesized copolymer was properly filtered off and subsequently washed with adequate amount of $1 \mathrm{M} \mathrm{HCl}$ and dried in oven for $8 \mathrm{~h}$ at $80^{\circ} \mathrm{C}$. An Emeraldine blackish-brown salt was obtained in each case.

\section{Characterization techniques used}

The solubility of the homopolymers and the copolymer samples was determined by dissolving $10 \mathrm{mg}$ of polymer in powdered form in the organic solvent such as in $\mathrm{N}$-methyl pyrrolidone (NMP), and was allowed to dissolve thoroughly in $100 \mathrm{~mL}$ of solvent. The dispersion is kept for few hours at the room temperature of $25{ }^{\circ} \mathrm{C}$; the polymer dispersion is now being filtered through sintered glass crucible (porosity 2 micron). UV-visible absorption spectra of dilute solutions of the polymer samples in NMP were recorded at room temperature on a Thermofisher scientific Genesys 10S UV-visible spectrophotometer, USA. Moreover, PerkinElmer 783/883 FTIR spectrophotometer, USA, was used to record the FTIR spectra of the samples in the form of $\mathrm{KBr}$ pellets. The electrical conductivity of the compressed pellets of the different polymer samples was recorded by the twoprobe method using a Keithley 617 electrometer. An Auto Hi-Res Q500, California, USA, and PerkinElmer Pyris, USA, were used to study the relative thermal stability of polymer by heating the polymer at a rate of $10{ }^{\circ} \mathrm{C} \mathrm{min}^{-1}$ under the nitrogen atmosphere. To investigate the surface topology of the materials, 
Nanoscope III SPM scanning probe atomic force microscope (AFM), USA, was employed using films of the polymers on silicon wafer. The dilute solution of few $\mu \mathrm{M}$ concentrations of the polymers in NMP solvent was prepared by sonication and centrifugation and dropped on silicon wafer for imaging AFM. Particle size distribution curve measured in NMP solvent by Malvern Instruments Ltd, Zetasizer ver. 7.10, UK.

\section{Results and discussion}

\section{Synthesis and yield}

The copolymers were synthesized using varying mole ratios of aniline and 3-nitroaniline monomers, as mentioned in Table 1. The chemical structure of the homopolymers and copolymer is shown in Fig. 1a, b. The yield of the copolymers is lower compared to PA but similar to that with P-3-NA as observed from Table 1. Even the yields of the different copolymers are comparable among themselves. This could be due to the highly electron-withdrawing nature of the nitro group $\left(-\mathrm{NO}_{2}\right)$ which reduces the electron density on the ring inhibiting oxidative polymerization process. Well-established oxidative polymerization mechanism suggests that oxidatively formed cation radicals get rearranged and two cation radicals add head-to-tail fashion at the para-position to form first dimer with release of protons; the chain propagation process eventually repeats leading to polymeric species [50]. Due to high electron-withdrawing nature of $-\mathrm{NO}_{2}$ group, 3-nitroaniline is less prone to oxidation compared to aniline, and thus, formation of the corresponding cation radical species is less favorable. Moreover, steric interferences of bulky $-\mathrm{NO}_{2}$ group considerably subdue the chain propagation step during oxidative polymerization. In addition, strong intramolecular/ intermolecular interaction through hydrogen bonding between the oxygen of the $-\mathrm{NO}_{2}$ group and the (-NH-) imine hydrogen on PA chains (Fig. 1c) can lead to the formation of favorable six-member chelate ring, which may localize the electron density on the ring leading to further decrease in the rate of polymerization, thereby decreasing the product \%-yield [51].

Table 1 Mole ratio of monomers taken, percent yield, solubility and electrical conductivity data of various sample polymers synthesized

\begin{tabular}{lllll}
\hline $\begin{array}{l}\text { Polymers derivatives } \\
\text { As HCl salt }\end{array}$ & $\begin{array}{l}\text { 3-Nitroaniline/aniline } \\
\text { mole ratio taken }\end{array}$ & $\begin{array}{l}\text { Polymeriza- } \\
\text { tion } \% \text { yield }\end{array}$ & $\begin{array}{l}\text { Solubility (W/V\%), } \\
(\mathrm{g} / \mathrm{dL}), \mathrm{NMP}\end{array}$ & $\begin{array}{l}\text { DC electrical } \\
\text { conductivity } \\
\sigma,(\mathrm{S} / \mathrm{cm})\end{array}$ \\
\hline PA & 1 & 71 & $9.89 \times 10^{-2}$ & $5.60 \times 10^{-2}$ \\
PA-co-3-NA80 & $1: 4$ & 57 & $85.90 \times 10^{-2}$ & $1.53 \times 10^{-6}$ \\
PA-co-3-NA60 & $2: 3$ & 58 & $81.45 \times 10^{-2}$ & $1.85 \times 10^{-6}$ \\
PA-co-3-NA40 & $3: 2$ & 56 & $77.62 \times 10^{-2}$ & $2.51 \times 10^{-6}$ \\
PA-co-3-NA20 & $4: 1$ & 54 & $70.56 \times 10^{-2}$ & $3.46 \times 10^{-6}$ \\
P-3-NA & 1 & 60 & $89.67 \times 10^{-2}$ & $2.08 \times 10^{-7}$ \\
\hline
\end{tabular}


(A)

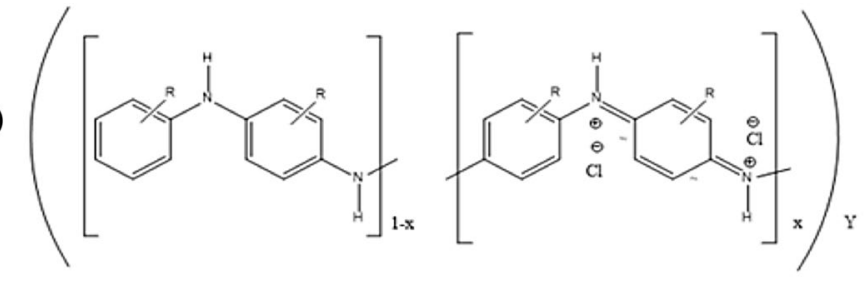

(B)

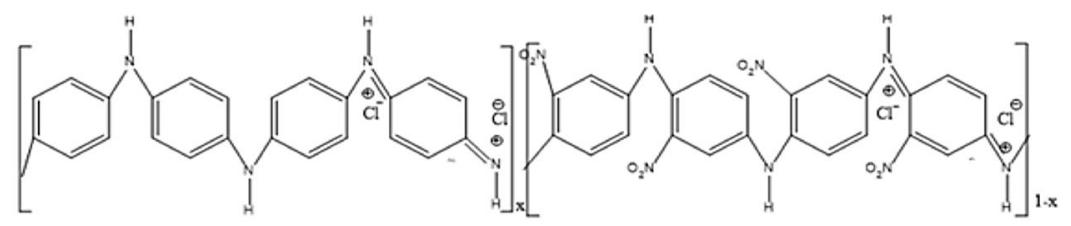

(C)

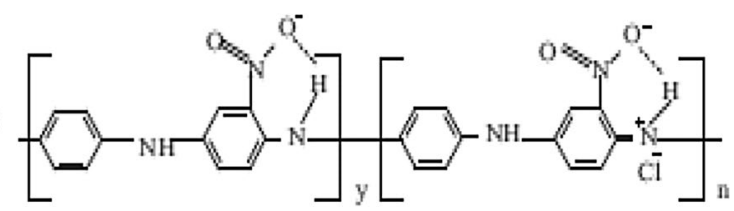

Fig. 1 Schematic representation of the general molecular structure of a homopolymers ( $\mathrm{R}=\mathrm{H}$ for aniline, $-\mathrm{NO}_{2}$ for 3-nitroaniline) and $\mathbf{b}$ for copolymers of aniline and 3-nitroaniline. $\mathbf{c}$ Schematic representation showing hydrogen bonded six-member chelate ring formation between the oxygen of the $-\mathrm{NO}_{2}$ group and hydrogen of the $-\mathrm{NH}$ imine group in the copolymer

\section{FTIR spectroscopic data analysis}

To ascertain the nature of functionalities present in the as-synthesized homopolymers and copolymer samples, FTIR spectra of PA, P-3-NA and doped copolymers with extreme compositions, i.e., PA-co-3-NA80, PA-co-3-NA20, respectively, are carried out and represented in Fig. 2. Neat PA shows characteristic peaks at 3608, 3447 (due to free and hydrogen bonded $\mathrm{N}-\mathrm{H}$ stretching frequencies), $1652\left(-\mathrm{NH}_{2}\right.$ bending vibration), and characteristic peaks at $1586 \mathrm{~cm}^{-1}$ and at $1522 \mathrm{~cm}^{-1}$ are assigned to the stretching vibrations of quinoid and benzenoid rings that play vital role in assigning the conductivity states in PA systems. It is also claimed that the highest conducting (metallic), emeraldine oxidation state of polyaniline contains 50\% of the nitrogen atoms in protonated state that consists of equal numbers of reduced, i.e., benzenoid diamine as well as oxidized, i.e., quinoid diamine repeat units in the polymer skeleton [52-54]. In the present case, optimum protonation of the imine nitrogen and improved chain alignment of polymer strands leads to the ideal ratio of intensities of these peaks, indicating the presence of emeraldine form of PA. This is further supported by the band near $1351 \mathrm{~cm}^{-1}$ and is assigned to the $\mathrm{C}=\mathrm{N}+$ stretching adjacent to the quinoid structure, and a medium intensity band at $1310 \mathrm{~cm}^{-1}$ is associated with $\mathrm{C}-\mathrm{N}$ stretching vibration in the alternate units of quinoid-benzenoid-quinoid rings, respectively. The band of conducting protonated form is observed at $1272 \mathrm{~cm}^{-1}$ while around $1165 \mathrm{~cm}^{-1}$ is attributed to $\mathrm{B}-\left(\mathrm{NH}^{+}\right)=\mathrm{Q}$ structure which is formed during the protonation process. 


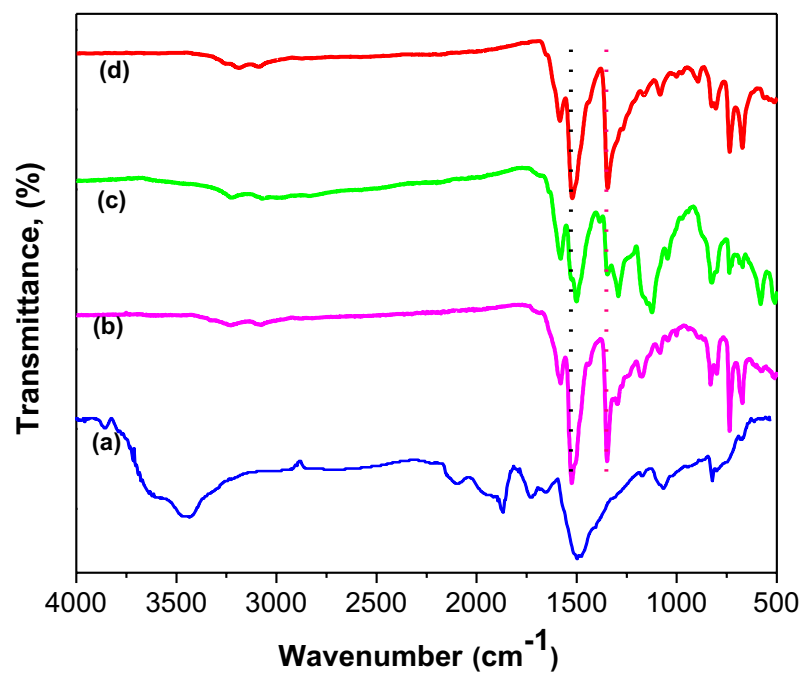

Fig. 2 FTIR spectra of (a) PA, (b) PA-co-3-NA80, (c) PA-co-3-NA20 and (d) P-3-NA

In addition, the peak at $675 \mathrm{~cm}^{-1}$ is attributed to $-\mathrm{NH}_{2}$ wagging and the band at $1090 \mathrm{~cm}^{-1}$ is assigned to the $\mathrm{C}-\mathrm{H}$ in plane bending, respectively. Thus, the above assigned peaks designate the presence of emeraldine salt form of PA in accordance with previous literature reports [51, 55, 56]. In P-3-NA, additional bands characteristic of asymmetric and symmetric stretching vibrations of $-\mathrm{NO}_{2}$ group is located at around $1530 \mathrm{~cm}^{-1}$ and $1335 \mathrm{~cm}^{-1}$, respectively. The spectra of the copolymers show the main bands in the region of $1590 \mathrm{~cm}^{-1}, 1508 \mathrm{~cm}^{-1}$ and $1308 \mathrm{~cm}^{-1}$ corresponding to the ringstretching vibrations of the quinoid and benzenoid rings of nitroaniline, respectively. The peaks at $1292 \mathrm{~cm}^{-1}$ and $1210 \mathrm{~cm}^{-1}$ correspond to $\mathrm{N}-\mathrm{H}$ bending and the symmetric component of the $\mathrm{C}-\mathrm{C}$ (or $\mathrm{C}-\mathrm{N}$ ) stretching modes, respectively [57]. The band around $1170 \mathrm{~cm}^{-1}$ is attributed to $\mathrm{B}-\left(\mathrm{NH}^{+}\right)=\mathrm{Q}$ structure which is formed as a result of copolymer protonation in acid medium. The bands $\sim 825 \mathrm{~cm}^{-1}$ can be attributed to out-of-plane $\mathrm{C}-\mathrm{H}$ bending modes, respectively. The significant broadening along with intensity lowering of the frequencies around $3000 \mathrm{~cm}^{-1}$ can be related to the hydrogen bonds formed between the oxygen of the nitro group and the hydrogen of the amine group (Fig. 1c), in agreement with similar reports Moreover, the intensity of the relative peak for asymmetric and symmetric stretching vibrations of $-\mathrm{NO}_{2}$ group at around $1530 \mathrm{~cm}^{-1}$ lies in the order: P-3NA > PA-co-3-NA80 > PA-co-3-NA20, subsequently confirming the formation of the desired copolymers.

\section{Comparative study of solubility of the homopolymers and copolymers}

As already stated PA has vivid potential applications, but the major problem in employing these systems commercially is due to their poor processibility by solvent. Most of them have exceedingly low solubility in common solvents. Copolymerization offers a way of improving the processibility of PA [58]. The poor solubility of PA in common solvents is due to strong cohesive interaction through $\pi-\pi$ 
interactions and strong interchain hydrogen bonding. On the other hand, P-3-NA exhibits higher solubility, owing to its side substituent $-\mathrm{NO}_{2}$ (also polar group) that reduces these interchain cohesive interactions considerably. Thus, introduction of 3-NA segments in the skeleton of PA in the copolymer increases the affinity for dissolution in common organic solvents like NMP as observed in Table 1. Thus, the solubility order stands as P-3-NA $\gg$ PA-co-3-NA80 $>$ PA-co-3-NA60 $>$ PA-co3-NA40 > PA-co-3-NA20 $\gg$ PA in NMP solvent at $25^{\circ} \mathrm{C}$.

\section{Electrical conductivity comparative study}

Table 1 highlights the comparative study of the DC electrical conductivity of the pristine as well as copolymer samples, measured at room temperature. It is evident that the DC conductivity of the copolymer increases with increasing concentration ratio of aniline to 3-nitroaniline in the copolymer. The values are considerably lower than that of PA but greater than P-3-NA. The higher conductivity of PA exhibited is because of the existence of emeraldine salt form as evident from the FTIR studies. Moreover, the presence of dopant ion $(\mathrm{Cl})$ as evident from EDX study, Supporting Information-1A, further supports the formation of the emeraldine salt form of PA. The quinoid-to-benzenoid peak intensity ratios as revealed from FTIR studies are 1.00 (PA), 1.14 (P-3-NA), 1.02 (PA-co-3-NA20) and 1.09 (PA-co-3-NA80), respectively. However, this ratio is nearly similar for other samples too, but still a large variation in conductivity is observed. Even the doping level is similar to that of PA as evident from EDX study, Supporting Information-1B.

It has been recently proved that parallel alignment of polymeric chains is very crucial for superior charge transport properties [54]. Thus, low conductivity in copolymers and P3NA may be attributed to the presence of $-\mathrm{NO}_{2}$ group in the PA segments that disrupts the polymer chain alignment; as a result, the $\pi$-conjugation is hindered, which in turn inhibits long-range $\pi$-electron delocalization and thus decreases the charge transport characteristics. Again, increased separation of the copolymer chain due to the presence of side groups along with the presence of electron-withdrawing $-\mathrm{NO}_{2}$ group in the copolymer chain decreases the interchain charge transport that further lowers the electrical conductivity. Thus, increasing substitution in the copolymers experiences lower charge transport is in accordance with the reports obtained from the literature [49, 57-59]. So, tuning of degree of substitution in these systems is very important. Moreover, favorable six-member chelate ring formation through strong intermolecular as well as intramolecular hydrogen bonding interactions between $-\mathrm{NO}_{2}$ groups and the polaronic nitrogen atoms of imine group in PA skeleton as discussed in the previous section (Fig. 1c) promotes localized structure resulting in a lowering electrical conductivity as well. Consequently, the electrical conductivity order stands as PA $\ggg$ PA-co-3-NA20 $>$ PA-co3-NA40 $>$ PA-co-3-NA60 $>$ PA-co-3-NA80 $\gg$ P-3-NA at room temperature. Thus, the electrical conductivity in P-3-NA as well as in these copolymers is thus dominated by the extent of $-\mathrm{NO}_{2}$ group present therein the polymer chains. Therefore, we infer that tuning the proportions of $-\mathrm{NO}_{2}$ groups as well as quinoid-to-benzenoid 


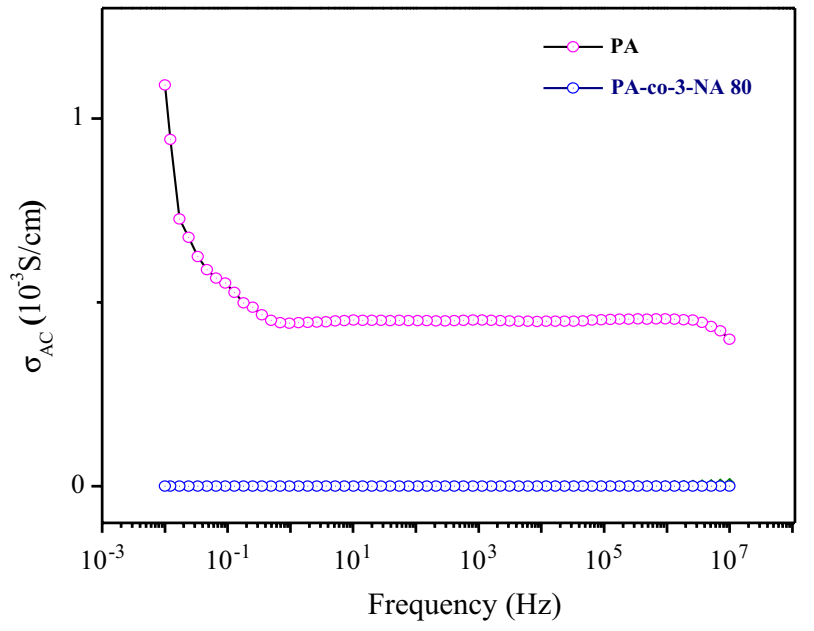

Fig. 3 Comparative study of AC conductivity of PA and PA-co-3-NA80 within the frequency range of $10^{-3}$ to $10^{8} \mathrm{~Hz}$, measured at room temperature

ratio are essentially to be tuned simultaneously so as to achieve desirable electrical conductivity in these copolymers.

\section{AC conductivity}

Figure 3 displays the comparative study of the conductivity impact of homopolymer PA and copolymer PA-co-3-NA80 with varying frequency range from $10^{-3}$ to $10^{8} \mathrm{~Hz}$ at room temperature. The conductivity of PA is observed to be higher than the copolymer. This is attributed to incorporation of bulky $-\mathrm{NO}_{2}$ group onto the polymer backbone in the latter. This leads to break in $\pi$-conjugation as the alignment of polymer chains get disrupted and entangled on substitution with such bulky groups that causes alteration in the HOMO-LUMO energy band gap. It has also been observed that optimum proportions of side groups can, however, modify and lower the HOMO-LUMO band energy gap leading to increased conductivity compared to pristine PANI as discussed for PA-co-3-NA20 sample in our previous work [59]. Thus, it is obvious that choice of proper composition is essential in tuning the conductivity in these copolymers.

\section{Dielectric measurements}

Figure $4 \mathrm{a}, \mathrm{b}$ shows relative variations of $\varepsilon^{\prime}$ and $\varepsilon^{\prime \prime}$ against a range of frequency at room temperature of PA and PA-co-3-NA80 samples, respectively. In both samples, at low frequency, both $\varepsilon^{\prime}$ and $\varepsilon^{\prime \prime}$ have high magnitude but becomes almost invariant at higher frequencies. Such signature confers that interfacial polarization contribute mostly at lower frequencies, while at higher frequencies, the dipolar relaxation contribution predominates [60, 61]. In HCl-treated PA, doping effect generates 

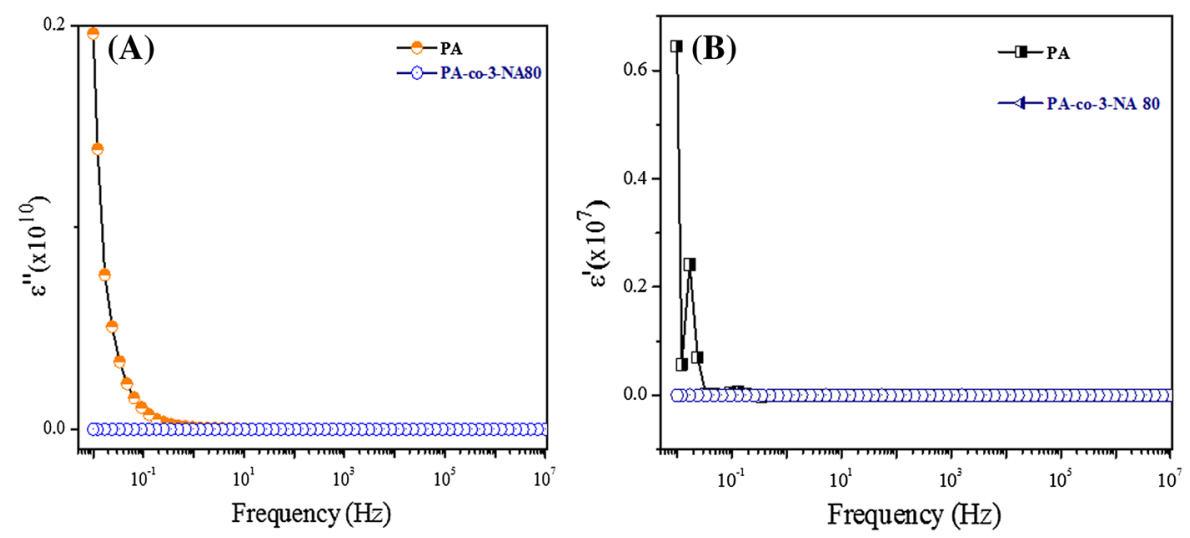

Fig. 4 a, b Relative variations of $\varepsilon^{\prime}$ and $\varepsilon^{\prime \prime}$ against a range of frequency at room temperature of PA and PA-co-3-NA80 samples

polarons/bipolarons that trigger charge delocalization along the polymer chain lengths, which augments the interfacial polarization resulting in large dielectric responses, well in agreement with previous reports [62]. However, at high frequencies, rapid periodic reversal of electric field restricts ion diffusion that diminishes the interfacial polarization, thus causing decrease in response. Doping is present in both PA-co-3-NA80 and pristine PA (EDX study, Supporting Information-1A-B). EDX of copolymer shows relatively more intense peaks for $\mathrm{Cl}$ (dopant ion) and is expected to exhibit higher dielectric signature. However, the bulky $-\mathrm{NO}_{2}$ groups considerably restrict proper orientation of the polymer chains, leading to low value. Thus, overall dielectric signature for the PA and copolymer is similar except the fact that the copolymers exhibit intermediate dielectric values at lower frequency compared to the homopolymers. It is worth notifying that the observed dielectric features in these systems are superior to various other conducting polymers complexes based on MEH-PPV hybrid systems as well as conducting polymer-based composite systems [63-65], which makes these systems interesting materials for next-generation organo-electronics.

\section{Thermogravimetric analysis}

Figure 5 shows the TGA profiles of all the PA, P-3-NA and copolymer samples. The initial mass loss up to $200{ }^{\circ} \mathrm{C}$ is negligible for P-3-NA homopolymer compared to about $14 \%$ for PA homopolymer attributed to loss of adsorbed water and moisture content in the samples [66]. Beyond $200{ }^{\circ} \mathrm{C}$, PA undergoes another large weight loss at $280^{\circ} \mathrm{C}$ owing to cleavage of intermolecular hydrogen bonds, carbon skeleton and spontaneously degrades to $25 \%$ of the initial mass at $800{ }^{\circ} \mathrm{C}$ (with mass loss $\sim 60 \%$ at this step). However, in P-3-NA, two-step decomposition occurs at $260{ }^{\circ} \mathrm{C}$ and at $400{ }^{\circ} \mathrm{C}$, respectively, showing an overall mass loss of $\sim 63 \%$ at $800{ }^{\circ} \mathrm{C}$. The higher thermal stability of P-3-NA compared to PA indicates appreciable intermolecular interactions (hydrogen bonding between $-\mathrm{NO}_{2}$ and $-\mathrm{NH}$ groups on $\mathrm{PA}$ 


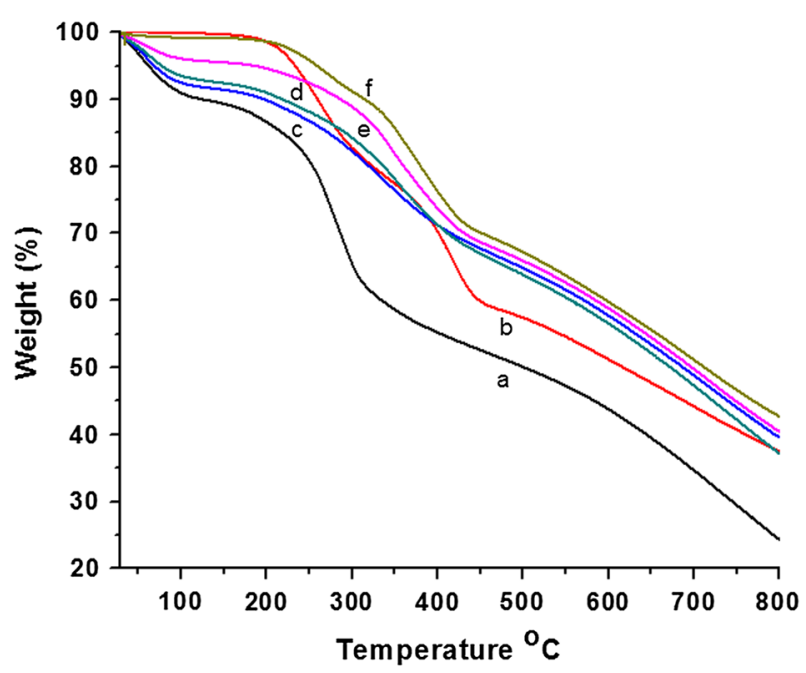

Fig. 5 TGA profile of (a) PA, (b) P-3-NA, (c) PA-co-3-NA80, (d) PA-co-3-NA60, (e) PA-co-3-NA40 and (f) PA-co-3-NA20

skeleton) (Fig. 1c) induced by the nitro group substituents [67]. The gradual loss $\left(260{ }^{\circ} \mathrm{C}\right.$ for P-3-NA) is assigned to some morphological change, possibly crystallization of the polymers due to annealing condition followed by subsequent loss of dopants and decomposition of the polymer material with rising temperatures. The nitro group in the copolymers introduces aforesaid additional interactions bringing the polymer chains together, thereby imparting stronger cohesive forces that lead to extra thermal stability. In the present case, PA-co-3-NA20 shows such optimized cohesive interactions. However, with the increasing proportion of bulky $-\mathrm{NO}_{2}$ groups, segregation of PA and P-3-NA phases occurs that widens the interchain separation leading to inverse effect. The above justification supports the observed increasing order of thermal stability and thus stands as PA $\ll$ PA-3-NA $<$ PA-co3 -NA80 < PA-co-3-NA60 < PA-co-3-NA40 < PA-co-3-NA20.

\section{UV-Vis spectroscopic data analysis}

Figure 6 shows the UV-visible spectra of the pristine PA, P-3-NA and its copolymer samples. PA is reported to exhibit peak at $280-295 \mathrm{~nm}$ due to the $\pi-\pi^{*}$ transition of the benzenoid ring associated with the extent of conjugation between the adjacent phenyl rings in the polymer chain [68]. The peak at $605-635 \mathrm{~nm}$ is attributed to the quinoid ring transition (charge transfer from HOMO of the benzenoid ring to LUMO of the quinoid ring) and is dependent on the overall oxidation state of the polymer. A characteristic band for polaron- $\pi^{*}$ transition appeared at $450 \mathrm{~nm}$, indicating that the resulting PA emeraldine salt was in the doped state, which further supports the findings from FTIR studies $[68,69]$. In our sample, the intermediate peak at $331 \mathrm{~nm}$ is attributed from the contribution of overlapping peaks of both $\pi-\pi^{*}$ transition and polaron- $\pi^{*}$ transition, respectively. In P-3-NA, the presence of $-\mathrm{NO}_{2}$ substituent 


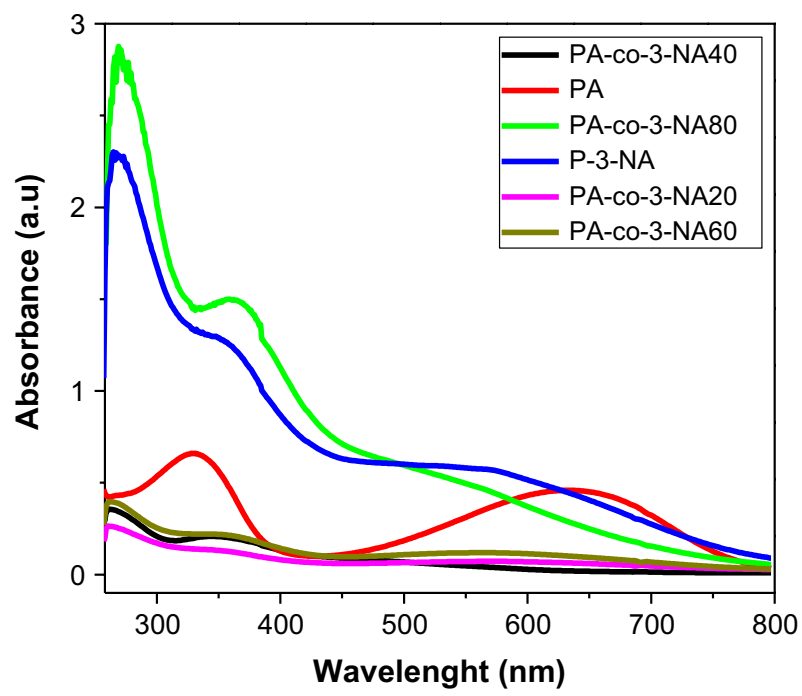

Fig. 6 UV spectra of P-3-NA, PA, PA-co-3-NA80, PA-co-3-NA60, PA-co-3-NA40 and PA-co-3-NA20 recorded in NMP solvent

causes entangling of polymer chains that disrupt the conjugation length and leads to hypsochromic shift of $\pi-\pi^{*}$ transition at around $270 \mathrm{~nm}$, polaron- $\pi^{*}$ transition at $\sim 352 \mathrm{~nm}$ and quinoid ring transition to $\sim 550 \mathrm{~nm}$, respectively. In the copolymers, due to random substitution of the monomers, the conjugation length is further shortened that results in further hypsochromic shift of $\pi-\pi^{*}$ transition to $263 \mathrm{~nm}$. The polaron- $\pi^{*}$ transition as well as the quinoid ring transition is highly influenced by the extent of intrinsic doping of PA with 3-NA residues and accordingly shows peak shifts for the copolymer samples. PA-co-3NA80 shows obvious similar features with P-3-NA due to the presence of greater proportion of 3-NA segments. Thus, the study narrates that compared to homopolymers; the copolymers absorb energy almost throughout the full range of visible region of energy spectrum (200-800 nm) and are thus obviously going to play important role in polymer-based solar cell applications. Again, the solubility of the copolymers varies: PA-co-3NA60 > PA-co-3NA40> PAco-3NA20 and so absorbance will vary accordingly. Thus, the above study indicates that controlled incorporation of $-\mathrm{NO}_{2}$ groups moieties onto PA segments appreciably modifies the optoelectronic structure in the resultant copolymers.

\section{AFM analysis (morphology study)}

The topography of surface of the polymer samples of PA, P-3-NA and the PA-co3NA60 copolymer at nanometer scale was studied using AFM as shown in Fig. 7. The dimension of the film used in this case is $(3 \times 3) \mu \mathrm{m}^{2}$ produced on drop casting from NMP solution. The surface profile of PA indicates non-uniform surface of the polymer. Such poor quality film formation results from poor dispersion in 
(A)

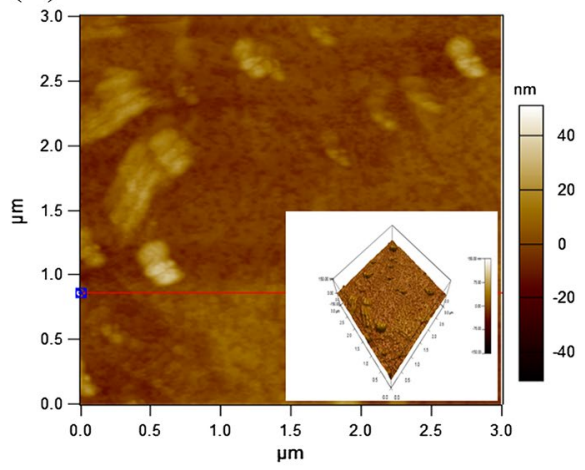

(B)

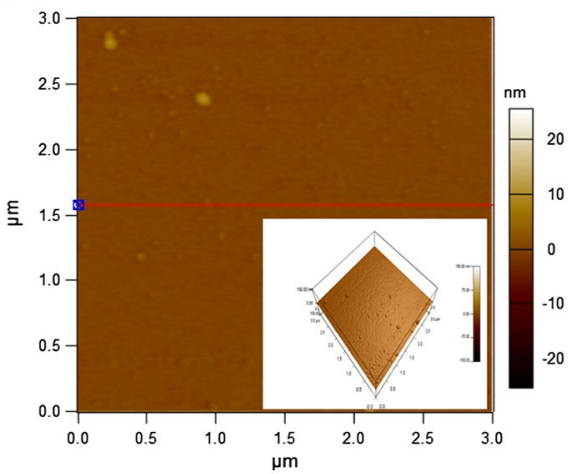

(C)

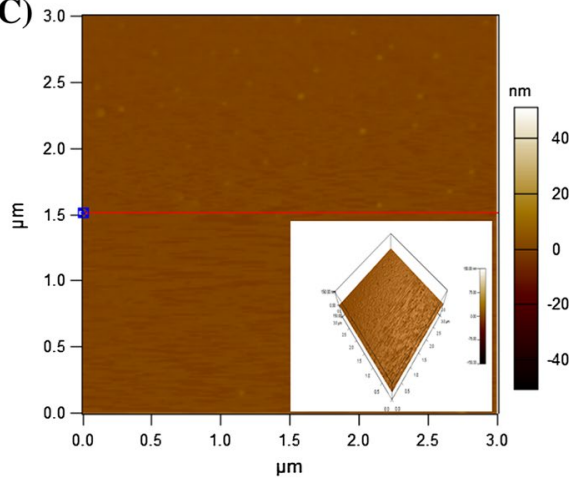

Fig. 7 AFM images of a PA, b P-3-NA and $\mathbf{c}$ PA-co-3-NA60

solution. On the contrary, better dispersion leads to smoother and more uniform, continuous and defect-free surface characteristics in P-3-NA as well as copolymer PA-co-3NA60. Such superior smooth surface film formation through simplistic route recommends their applications in the challenging areas of device fabrication for chemical sensing, bio-sensing and other superior electronic devices [70-73].

\section{Particle size analysis}

Particle average-size determination is crucial for correlating various physicochemical properties of systems. Figure $8 \mathrm{a}-\mathrm{c}$ shows the particle size distribution curves of polyaniline PA, P-3-NA and the PA-co-3NA60 copolymer in NMP solvent, respectively. For PA, the curve indicates that the particle size varies in the range 5-9000 $\mathrm{nm}$ and a small fraction of particles possess a size in the range of 5-10 $\mathrm{nm}$. A large fraction of polyaniline possesses the particle size in the range of $100-1000 \mathrm{~nm}$. An average particle size varies in the range of 1000-9000 $\mathrm{nm}$. For P-3-NA, the particle size varies in the range 100-1000 nm and 2000-8000 nm with larger fraction in the latter range. The PA-co-3NA60 copolymer shows a large fraction of the sample in the 5000-8000 $\mathrm{nm}$ range and a small fraction of 


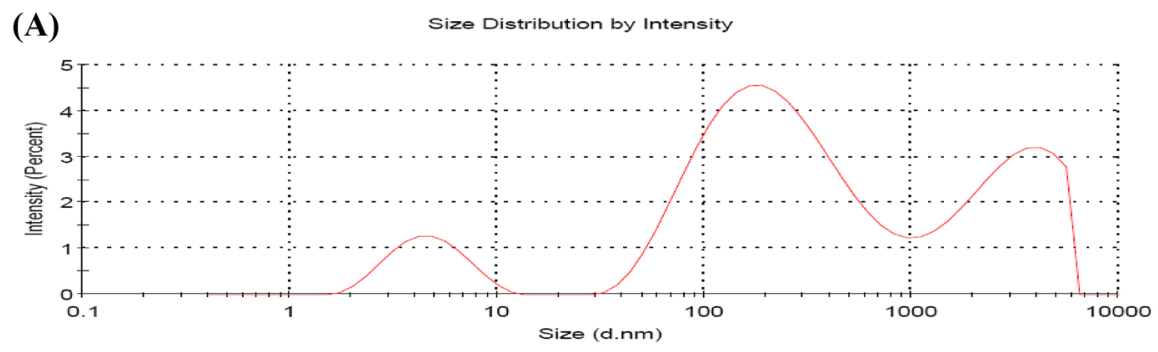

(B)

Size Distribution by Intensity

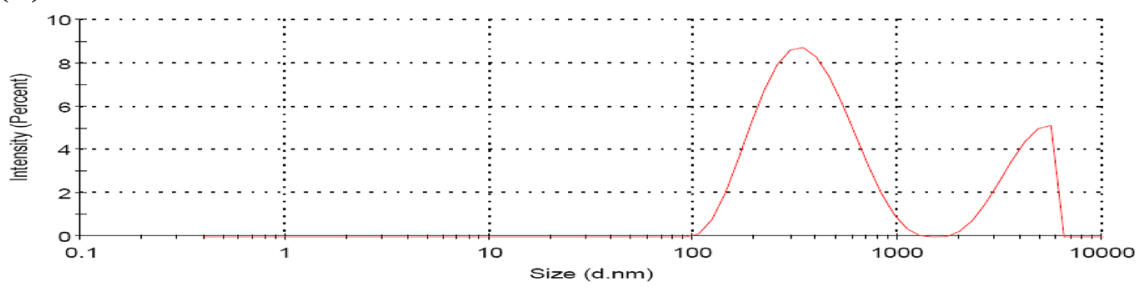

(C)

Size Distribution by Intensity

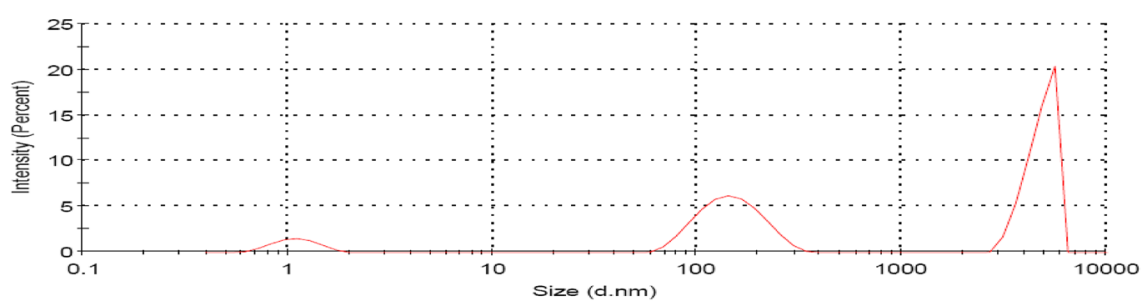

Fig. 8 Particle size distribution curve of a PA, b P-3-NA and c PA-co-3-NA60

the particles lie in less than $5 \mathrm{~nm}$ and $80-500 \mathrm{~nm}$ ranges. The particle size varies on account of the degree of $-\mathrm{NO}_{2}$ functional groups present in polyaniline segments. Owing to the larger proportions of bulky $-\mathrm{NO}_{2}$ substituents in the copolymer, the particle sizes vary in the range of 5000-8000 nm under the observed conditions with narrow range of distribution. It can be realized that owing to the dominance of narrow range of particle size in PA-co-3NA60, the copolymer film adopted smoother morphology as revealed in AFM studies and thus ideally suited for improving film-fabrication technology associated with sensors, optoelectronic and energy storage applications [56-59].

The average particle size distribution as it stands as PA $<$ P-3-NA $<$ PA-co-3NA60 also can be well correlated with their thermal properties too. Since the copolymer sample possesses larger average particle size value compared to their homopolymers, the heat of degradation is higher, leading to improved thermal stability. The improved results are in well agreement with that expected from DFT studies carried out by other workers [30]. 


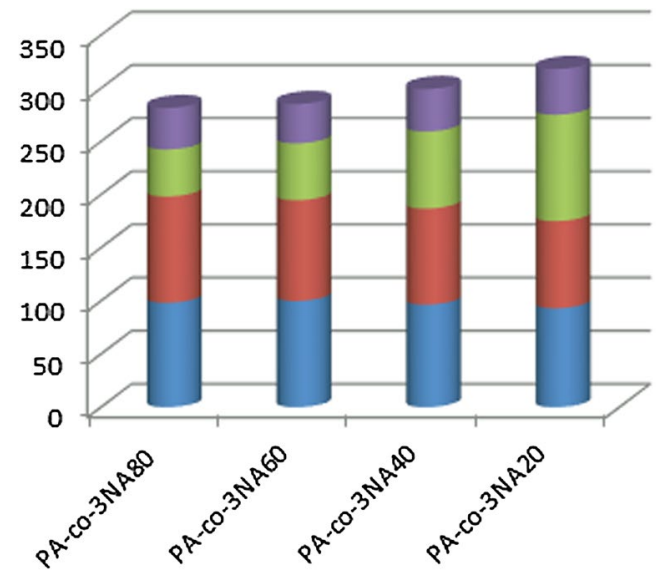

- RELATNE PERCENTAGE

THERMAL STABILITY

RELATNE PERCENTAGE CONDUCTIITY

RELATNE PERCENTAGE SOLUBILITY

RELATNE PERCENTAGE \%YIELD

Fig. 9 Statistical survey of the relative percentage with respect to the yield, solubility, solubility in NMP solvent and thermal stability at $800{ }^{\circ} \mathrm{C}$, respectively, of the copolymers of different compositions

Thus, from the above-studied physicochemical trends explored for all the as-synthesized copolymer samples of varied compositions, a statistical survey of the relative percentage with respect to the yield, solubility, solubility in NMP solvent and thermal stability at $800{ }^{\circ} \mathrm{C}$, respectively, was carried out and is displayed in Fig. 9. The results inferred that the PA-co-3NA20 sample possesses the optimized properties among all and is therefore ideally suitable for multifunctional applications.

\section{Conclusions}

Thus, we report here a series of copolymers of aniline and 3-nitroaniline that are chemically synthesized by varying the molar ratio of the monomers via oxidative copolymerization reaction. The characterization studies confirm the presence of both the monomer units employed in the copolymer formation for the all compositions presently studied. The solubility in polar organic solvent and the charge transport of copolymers can be effectively modified and optimized accordingly by varying the monomer composition through controlling the proportion of $\left(-\mathrm{NO}_{2}\right)$ functional groups as substituents in the polymer chains. Dielectric measurements conferred that the degree of functionalization of side groups $\left(-\mathrm{NO}_{2}\right)$ essentially regulates polarization behavior in the derived polymers. The copolymers also exhibited higher thermal stability attributed to larger yet narrow average particle size possession compared to the homopolymers. Moreover, the copolymers manifest smoother surface film formation compared to PA or P-3-NA homopolymers, ideally suited for smart device fabrication. Thus, the above features achieved through copolymerization make the material enormously potential for applications for the fast-developing areas of organo-based photovoltaics, energy storage, sensing and allied technological fields. 
Acknowledgements The authors are thankful to the Qatar University. DM further acknowledges Chandernagore College, Hooghly, WB, India-712136, for giving permission to do honorary research.

Open Access This article is distributed under the terms of the Creative Commons Attribution 4.0 International License (http://creativecommons.org/licenses/by/4.0/), which permits unrestricted use, distribution, and reproduction in any medium, provided you give appropriate credit to the original author(s) and the source, provide a link to the Creative Commons license, and indicate if changes were made.

\section{References}

1. Patil AO, Heeger AJ, Wudl F (1988) Optical properties of conducting polymers. Chem Rev 88:183-200

2. Heeger AJ, Kivelson S, Schrieffer JR, Su WP (1988) Solitons in conducting polymers. Rev Mod Phys 60:781

3. Brabec CJ, Sariciftci NS, Hummelen J (2001) Plastic solar cells. Adv Funct Mater 11:15-26

4. Xia L, Wei Z, Wan M (2010) Conducting polymer nanostructures and their application in biosensors. J Colloid Interface Sci 341:1-11

5. Wang Y, Jing X (2005) Intrinsically conducting polymers for electromagnetic interference shielding. Polym Adv Technol 16:344-351

6. Das TK, Prusty S (2012) Review on conducting polymers and their applications. Polym Plast Technol Eng 511:487-1500

7. Angelopoulos M (2001) Conducting polymers in microelectronics. IBM J Res Dev 45:57-75

8. Macdiarmid AG, Chaiang JC, Richter AF, Epstein AJ (1987) Polyaniline: a new concept in conducting polymers. Synth Met 18:285-290

9. Boeva Z, Sergeyev V (2014) Polyaniline: synthesis, properties, and application. Polym Sci Ser C 56:144-153

10. Wang ZH, Li C, Scherr EM, MacDiarmid AG, Epstein AJ (1991) Three dimensionality of "metallic', states in conducting polymers: polyaniline. Phys Rev Lett 66:1745-1748

11. Tas R, Can M, Sonmezoglu S (2015) Preparation and characterization of polyaniline microrods synthesized by using dodecylbenzene sulfonic acid and periodic acid. Turk J Chem 39:589-599

12. Sönmezoğlu S, Taş R, Akın S, Can M (2012) Polyaniline micro-rods based heterojunction solar cell: structural and photovoltaic properties. Appl Phys Lett 101:253301

13. Taş R, Tohumcu C, Can M (2014) Increasing the crystallite and conductivity of polypyrrole with dopant used. Ionics 20(12):1687-1692

14. Majumdar D, Maiti RP, Basu S, Saha SK (2009) Mechanism of ultrasonic energy-assisted formation of V-, Y-shaped nano-structures in conjugated polymers. J Nanosci Nanotechnol 9(12):6896-6901

15. Majumdar D (2019) Polyaniline as proficient electrode material for supercapacitor applications: PANI nanocomposites for supercapacitor applications. In: Ramdani N (ed) Polymer nanocomposites for advanced engineering and military applications. IGI Global Publishers, Pennsylvania. https:// doi.org/10.4018/978-1-5225-7838-3.ch007

16. Green RA, Lovell NH, Wallace GG, Poole-Warren LA (2008) Conducting polymers for neural interfaces: challenges in developing an effective long-term implant. Biomaterials 29:3393-3399

17. Balint R, Cassidy NJ, Cartmell SH (2014) Conductive polymers: towards a smart biomaterial for tissue engineering. Acta Biomater 10:2341-2353

18. Naveen MH, Gurudatt NG, Shim YB (2017) Applications of conducting polymer composites to electrochemical sensors: a review. Appl Mater Today 9:419-433

19. Spitalsky Z, Tasis D, Papagelis K, Galiotis C (2010) Carbon nanotube-polymer composites: chemistry, processing, mechanical and electrical properties. Prog Polym Sci 35:357-401

20. Zhuang XD, ChenY Liu G, Li PP, Zhu CX, Kang ET, Noeh KG, Zhang B, Zhu JH, Li YX (2010) Conjugated-polymer-functionalized graphene oxide: synthesis and nonvolatile rewritable memory effect. Adv Mater 22:1731-1735 
21. Batool A, Kanwal F, Imran Md, Jamil T, Siddiqi SA (2012) Synthesis of polypyrrole/zinc oxide composites and study of their structural, thermal and electrical properties. Synth Met 16:12753-12758

22. Coakley KM, McGehee MD (2004) Conjugated polymer photovoltaic cells. Chemistry of Materials 16:4533-4542

23. Dakshayini BS, Reddy KR, Mishra A, Shetti NP, Malode SJ, Basu S, Naveen S, Raghu AV (2019) Role of conducting polymer and metal oxide-based hybrids for applications in ampereometric sensors and biosensors. Microchem J 147:7-24

24. Reddy KR, Hemavathi B, Balakrishna GR, Raghu AV, Naveen S, Shankar MV (2019) 11: Organic conjugated polymer-based functional nanohybrids: synthesis methods, mechanisms and its applications in electrochemical energy storage supercapacitors and solar cells. In: Pielichowski K, Majka TM (eds) Micro and nano technologies, polymer composites with functionalized nanoparticles. Elsevier, Amsterdam, pp 357-379, ISBN No. 978-0-12-814064-2

25. Reddy KR, Jeong HM, Lee Y, Raghu AV (2010) Synthesis of MWCNTs-core/thiophene polymersheath composite nanocables by a cationic surfactant-assisted chemical oxidative polymerization and their structural properties. J Polym Sci A Polym Chem 48:1477-1484

26. Majumdar D (2019) Polyaniline nanocomposites: innovative materials for supercapacitor applications-PANI nanocomposites for supercapacitor applications. In: Ramdani N (ed) Polymer nanocomposites for advanced engineering and military applications. IGI Global Publishers, Pennsylvania. https://doi.org/10.4018/978-1-5225-7838-3.ch008

27. Majumdar D, Baskey M, Saha SK (2011) Epitaxial growth of crystalline polyaniline on reduced graphene oxide. Macromol Rapid Commun 32:1277-1283

28. Majumdar D (2016) Functionalized-graphene/polyaniline nanocomposites as proficient energy storage material: an overview. Innov Ener Res 5:145

29. Elsenbaumer RL, Jen KY, Oboodi R (1986) Processible and environmentally stable conducting polymers. Synth Met 15:169-174

30. Araujo MH, Matencio T, Donnici CL, Calado HDR (2016) Synthesis and electrochemical investigation of beta-substituted thiophene-based donor-acceptor copolymers with 3,4-ethylenedioxythiophene (EDOT). J Solid State Electrochem 20:2541-2550

31. Imae I, Sagawa H, Mashima T, Komaguchi K, OoyamaY Harima Y (2014) Synthesis of soluble polythiophene partially containing 3,4-ethylenedioxythiophene and 3-hexylthiophene by polycondensation. Open J Polym Chem 4:83-93

32. Imae I, Imabayashi S, Komaguchi K, Tan Z, OoyamaY Harima Y (2014) Synthesis and electrical properties of novel oligothiophenes partially containing 3,4-ethylenedioxythiophenes. RSC Adv 4:2501-2508

33. Göker S, Hizalan G, Udum YA, Toppare L (2014) Electrochemical and optical properties of 5,6-bis(octyloxy)-2,1,3 benzooxadiazole containing low band gap polymers. Synth Met 191:19-27

34. Wang X, Liu P (2014) Improving the electrochemical performance of polyaniline electrode for supercapacitor by chemical oxidative copolymerization with p-phenylenediamine. J Ind Eng Chem 20:1324-1331

35. Basavaraja C, Ho Jung G, Huh DS (2017) Morphology and electron transport property of polyaniline/poly(3,4-ethylenedioxythiophene) nanocomposite by the copolymerization of aniline and 3,4-ethylenedioxythiophene. Polym Compos 38:E261-E268

36. Waware US, Umare Suresh (2005) Chemical synthesis, spectral characterization and electrical properties poly(aniline-co-m-chloroaniline). React Funct Polym 65:343-350

37. Waware US, Hamouda AMS, Rashid M, Summers GJ (2017) The spectral and morphological studies of the conductive polyaniline thin film derivatives by the in situ copolymerization. J Mater Sci: Mater Electron 28:15178-15183

38. Waware US, Hamouda AMS, Shahul Hameed A, Summers Gabriel J (2017) Tuning the electrical properties of polyaniline by copolymerization with o-bromoaniline. Funct Mater Lett 10(1750039):1-6

39. Yalçınkaya S, Çolak N (2012) Synthesis and characterization of poly(aniline-co-o-aminoaniline). Des Monomers Polym 15(2):147-157

40. Rashid M, Sabir S (2009) Oxidative copolymerization of aniline with o- and p-nitroaniline by ammonium per sulfate: kinetic and pathway. J Dispers Sci Technol 30(3):297-304

41. Chen XP, Jiang JK, Liang QH, Yang N, Ye HY, Cai M, Shen L, Yang DG, Ren TL (2015) Firstprinciples study of the effect of functional groups on polyaniline backbone. Sci Rep 5:16907 
42. Von Oettingen WF (1941) The aromatic amino and nitro compounds, their toxicity and potential dangers. Public Health Bull 271:34

43. Pira E, Piolatto G, Negri E, Romano C, Boffetta P, Lipworth L, McLaughlin JK, La Vecchia C (2010) Bladder cancer mortality of workers exposed to aromatic amines: a 58-year follow-up. J Natl Cancer Inst 102:1096-1099

44. Dinga L, Li Q, Zhoua D, Cuia H, Tangb R, Zhai J (2012) Copolymerization of aniline with $\mathrm{m}$-nitroaniline and removal of m-nitroaniline from aqueous solutions using a polyaniline-modified electrode: a comparative study. Electrochim Acta 77:302-308

45. Ding L, Li Q, Zhou D, Cui H, Tang R, Zhai J (2012) Copolymerization of aniline with m-nitroaniline and removal of m-nitroaniline from aqueous solutions using a polyaniline-modified electrode: a comparative study. Electrochim Acta 77:302-308

46. Ibrahim KA (2017) Synthesis and characterization of polyaniline and poly(aniline-co-o-nitroaniline) using vibrational spectroscopy. Arab J Chem 10:S2668-S2674

47. Rashid M, Sabir Suhail (2009) Oxidative copolymerization of aniline with $o$ - and $p$-nitroaniline by ammonium per sulfate: kinetic and pathway. J Dispers Sci Technol 30:297-304

48. Roy BC, DuttaGupta M, Ray JK (1995) Studies on conducting polymers. 1. Aniline-initiated polymerization of nitroanilines. Macromolecules 28:1727-1732

49. Tran HD, D'Arcy JM, Wang Y, Beltramo PJ, Strong VA, Kaner RB (2011) The oxidation of aniline to produce "polyaniline": a process yielding many different nanoscale structures. J Mater Chem 21(11):3534-3550

50. Bhadra S, Singha NK, Khastgir D (2008) Effect of aromatic substitution in aniline on the properties of polyaniline. Eur Polym J 44(6):1763-1770

51. Kumar D (2000) Synthesis and characterization of poly(aniline-co-o-toluidine) copolymer. Synth Met 11:4369-4372

52. Salaneck WR, Lundström I, Huang W-S, MacDiarmid AG (1986) Electronic structure of some polyanilines. Synth Met 13:291

53. Ansari R, Keivani MB (2006) Polyaniline conducting electroactive polymers thermal and environmental stability studies. E-J Chem 3(4):202-217

54. Majumdar D, Saha SK (2015) Charge transport in polypyrrole nanotubes. J Nanosci Nanotechnol 15(12):9975-9981

55. Tzou K, Gregory RV (1993) A method to prepare soluble polyaniline salt solutions — in situ doping of PANI base with organic dopants in polar solvents. Synth Met 53:365-377

56. Yang Y, Mu S (2008) Synthesis and high electrochemical activity of poly(aniline-co-2-amino-4-hydroxybenzenesulfonic acid). Electrochim Acta 54:506-512

57. Rao PS, Anand J, Palaniappan S, Sathyanarayana DN (2000) Effect of sulphuric acid on the properties of polyaniline- $\mathrm{HCl}$ salt and its base. Eur Polym J 36:915-921

58. Han C-C, Hong S-P, Yang K-F, Bai M-Y, Lu C-H, Huang C-S (2001) Highly conductive new aniline copolymers containing butylthio substituent. Macromolecules 34:587-591

59. Waware US, Rashid M, Hamouda AMS (2018) Thermal stability and frequency-dependent electrical conductivity of poly(aniline-co-m-nitroaniline). Ionics. https://doi.org/10.1007/s1158 1-018-2741-X

60. Chutia P, Kumar A (2014) Electrical, optical and dielectric properties of $\mathrm{HCl}$ doped polyaniline nanorods. Phys B Condens Mater 436:200-207

61. Bekri-Abbes I, Srasra E (2015) Electrical and dielectric properties of polyaniline and polyaniline/ montmorillonite nanocomposite prepared by solid reaction using spectroscopy impedance. J Nanomater 16(1):428

62. Waware US, Hamouda AMS, Majumdar D (2018) Optimization of physicochemical and dielectric features in the conductive copolymers of aniline and 2-aminophenol. Polym Bull 1:2. https://doi. org/10.1007/s00289-018-2668-4

63. Brebels J, Manca J, Lutsen L, Vanderzande D, Maes W (2017) High dielectric constant conjugated materials for organic photovoltaics. J Mater Chem A 5:24037-24050

64. Rouis A, Davenas J, Bonnamour I, Ouada HB (2015) Studies of morphological optical and electrical properties of the MEH-PPV/azo-calix[4]arene composite layers. Phys B Condens Mater 474:70-76

65. Ahmed K, Kanwal F, Ramay SM, Mahmood A, Atiq S, Al-Zaghayer YS (2016) High dielectric constant study of $\mathrm{TiO}_{2}$-polypyrrole composites with low contents of filler prepared by in situ polymerization. Adv Condens Matter Phys 5:2. https://doi.org/10.1155/2016/47934

66. Ding L, Wang X, Gregory RV (1999) Thermal properties of chemically synthesized polyaniline EB powder. Synth Met 104:73-78 
67. Reddy KR, Raghu AV, Jeong HM (2008) Synthesis and characterization of novel polyurethanes based on 4,4'-\{1,4-phenylenebis[methylylidenenitrilo]\} diphenol. Polym Bull 60:609-616

68. Zainal MF, Mohd Y (2015) Characterization of PEDOT films for electrochromic applications. Polym Plast Technol Eng 54(3):276-281

69. Stejskal J, Kratochvíl P, Radhakrishnan N (1993) Polyaniline dispersions. 2. UV-Vis absorption spectra. Synth Met 61(3):225-231

70. Mahudeswaran A, Vivekanandan J, Vijayanand PS, Kojima T, Kato S (2016) A facile synthesis of poly(aniline-co-o-bromoaniline) copolymer: characterization and application as semiconducting material. Int J Mod Phys B 30(05):1650008

71. Mostafaei A, Zolriasatein A (2012) Synthesis and characterization of conducting polyaniline nanocomposites containing ZnO nanorods. Prog Nat Sci: Mater Int 22:273-280

72. Jaymand M (2013) Recent progress in chemical modification of polyaniline. Prog Polym Sci 38(9):1287-1306

73. Dedelaite L, Kizilkaya S, Incebay H, Ciftci H, Ersoz M, Yazicigil Z, Ramanavicius A (2015) Electrochemical determination of $\mathrm{Cu}(\mathrm{II})$ ions using glassy carbon electrode modified by some nanomaterials and 3-nitroaniline. Colloids Surf A 483:279-284

Publisher's Note Springer Nature remains neutral with regard to jurisdictional claims in published maps and institutional affiliations.

\section{Affiliations}

\section{Umesh Somaji Waware $^{1}$ - A. M. S. Hamouda ${ }^{1}$ - Dipanwita Majumdar ${ }^{2}$}

Umesh Somaji Waware

uswaware@gmail.com

$\triangle$ Dipanwita Majumdar wbesdmajumdar@gmail.com

1 Department of Mechanical and Industrial Engineering, College of Engineering, Qatar University, P. O. Box-2713, Doha, Qatar

2 Department of Chemistry, Chandernagore College, Hooghly, Kolkata, West Bengal 712136, India 